\title{
Polycapillary lenses for Soft X-Ray transmission in ITER: model, comparison with experiments and potential application ${ }^{\text {a) }}$
}

\author{
D. Mazon ${ }^{1, b)}$, C. Liegeard ${ }^{2}$, A.Jardin $^{1}$, R. Barnsley ${ }^{\text {c }}$, M. Walsh ${ }^{3}$, \\ M. O’Mullane ${ }^{4}$, A. Sirinelli ${ }^{3}$, F. Dorchies ${ }^{5}$ \\ ${ }^{1}$ CEA, IRFM F-13108 Saint-Paul-lez-Durance, France. \\ ${ }^{2}$ Ecole Polytechnique de Paris, France \\ ${ }^{3}$ ITER Organization, Saint-Paul-lez-Durance, France \\ ${ }^{4}$ Department of Physics SUPA, University of Strathclyde, Glasgow G4 ONG, UK \\ ${ }^{5}$ Univ. Bordeaux, CNRS, CEA, CELIA, UMR5107, Talence, 33405, France
}

\begin{abstract}
Measuring SXR radiation ([0.1 keV; $15 \mathrm{keV}])$ in tokamaks is a standard way of extracting valuable information on particle transport and magnetohydrodynamic activity. Generally, the analysis is performed with detectors positioned close to the plasma for a direct line of sight. A burning plasma, like the ITER Deuterium-Tritium phase, is too harsh an environment to permit the use of such detectors in close vicinity of the machine. We have thus investigated in this article the possibility of using polycapillary lenses in ITER, to transport the SXR information several meters away from the plasma in the complex portplug geometry.
\end{abstract}

(Presented XXXXX; received XXXXX; accepted XXXXX; published online XXXXX)

\section{INTRODUCTION}

\section{A. SXR measurements in tokamaks}

Tokamak plasmas emit as volumetric Soft X-Ray (SXR) sources and contain very useful information about plasma stability, shape and impurity content. Measuring the SXR radiation ([0.1 keV; $15 \mathrm{keV}])$ of magnetic fusion plasmas is thus a standard way of extracting valuable information on particle transport and magnetohydrodynamic activity. Generally, the analysis is performed with a 2D tomographic system composed of several cameras, equipped with detectors such as Silicon Barrier Diodes or Gas Electron Multipliers, positioned close to the plasma for a direct line of sight. A burning plasma, like the ITER Deuterium-Tritium phase, with high neutron fluxes, gamma and hard X-ray emission, high magnetic field and high radiofrequency powers, is too harsh an environment to permit the use of such detectors in close vicinity of the machine. We have thus investigated the possibility of using polycapillary lenses in ITER, to transport the SXR information several meters away from the plasma in the complex port-plug geometry. The idea is to protect the SXR detector from the entire environment using a proper shielding by positioning it far away from the plasma.

\section{B. Polycapillary lenses principles}

Polycapillary lenses or polycapillaries are optic devices made up with thin glass (borosilicate glass) capillaries. The capillaries are pipes whose diameters do not exceed few tens of micrometers. They are put together in order to guide X-rays and focus them on a selected point thanks to total reflections on the glass of each capillary, similarly to visible light in optical fibers. The main difference with visible light is that X-rays are easily absorbed by any solid materials during their propagation. Indeed, their reflection on any material is complicated by the fact that the wavelength has the same order of magnitude (between 0.06 and 1 $\mathrm{nm})$ as lattice constants of solid crystals $(\sim 0.1 \mathrm{~nm})$. Nonetheless, the index of refraction of most solids for X-rays is slightly inferior to 1, making possible for X-rays to reflect on solids in the air or in a vacuum without having a refracted wave. It is a total internal reflection. However, this reflection creates an evanescent wave in the solid from its surface (FIG. 1) that can lead to a loss of transmission. At each reflection, the reflection coefficient $R$ is calculated by the following equation depending on the energy $E$ of the photon and on the grazing angle $\theta_{\mathrm{i}}$ :

$$
R\left(\theta_{i}, E\right)=\left|\frac{\theta_{i}-\sqrt{\theta_{i}{ }^{2}-2 \delta(E)}}{\theta_{i}+\sqrt{\theta_{i}{ }^{2}-2 \delta(E)}}\right|^{2}<1
$$

with the complex index of refraction $n(E)=1-\delta(E)$

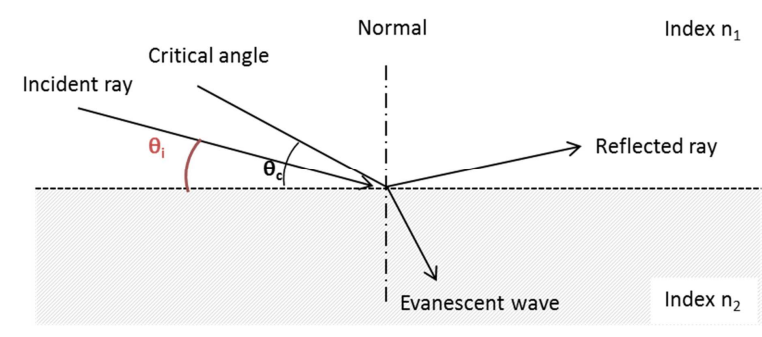

FIG. 1: X-ray reflection at a grazing incidence

Polycapillary lenses, depending on their structure, can guide a large beam, collimate it or focus it. Even if the transmission coefficient can be low for some cases, in tokamaks and particularly in ITER, the X-ray flow will be considerable (up to $250 \mathrm{~kW} / \mathrm{m}^{2}$ on the detectors) and even with huge losses (superior to $99.9 \%$ ), detectors will still be able to analyze the information carried by X-rays. 


\section{Model transmission and validation}

In order to develop a simulation tool for X-ray transmission through a single capillary, a code was developed starting from the theoretical principle of the photon path exposed in ${ }^{1}$. Full description of the model can be found in ${ }^{2}$. The simulations give good agreement with experimental data obtained at CELIA laboratory ${ }^{2}$. Protocol of the experiment can be found in ${ }^{2}$. Comparison between model and experimental data as well as influence of rugosity are presented in FIG. 2.

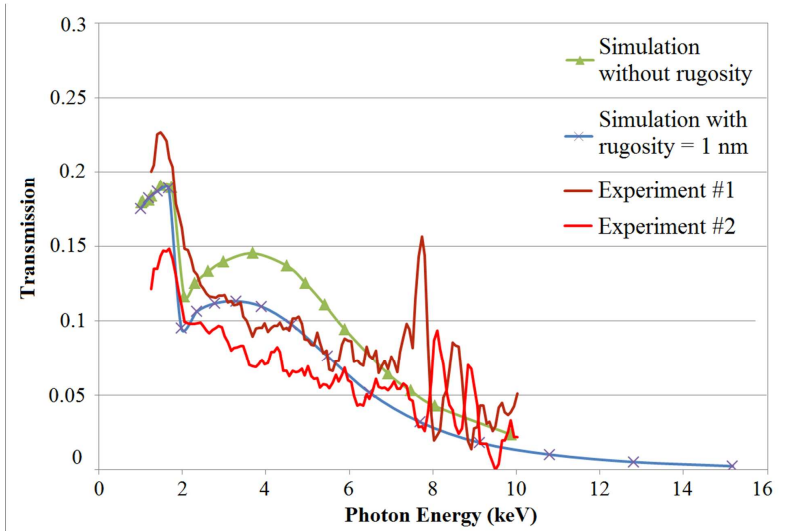

FIG. 2: Comparison model vs experimental results and influence of rugosity on the transmission

\section{ITER requirements}

The ITER team has already designed a volume box inside the port plug, see FIG. 3a-b, in which the SXR detectors are foreseen for the DD-phase: 6 beams with $\sim 10$ Lines of Sight $(\operatorname{LoS})$ for each beam are thus determined by this geometry (FIG. 3c).
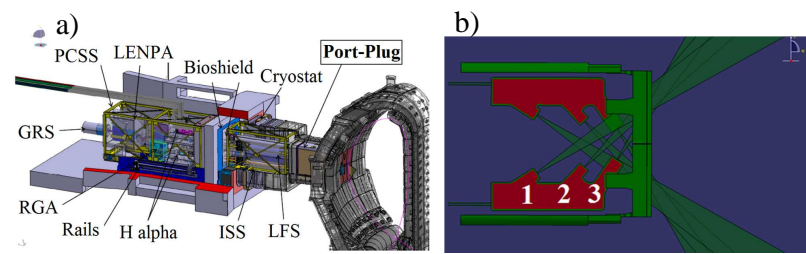

FIG. 3: a) Position of the port-plug (PP) compared with the containment chamber (source ITER), b) Upper and lower SXR beams and the space used by the diagnostics (in red). The exit of the port-plug is on the left
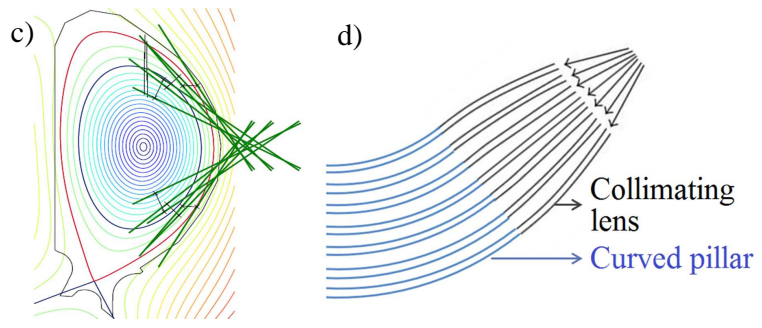

FIG. 3: c) SXR Lines of Sight (LoS) in the plasma limited by the red line (source ITER). d) Collimating lens + curved pillar
In the following part, we will only consider the box on the bottom of the port-plug due to the symmetry of the system. the beams are numbered from the left to the right from 1 to 3 in FIG. $3 \mathrm{~b}$. During the DT-phase the neutron flux will be $\sim 10^{10}$ neutrons.cm $\mathrm{c}^{-2} \cdot \mathrm{s}^{-1}$ on the detectors. Thus the detectors should be taken further away from the plasma, and the X-ray information (location of the source, energy and intensity) could be deflected to the detectors thanks to polycapillary lenses.

\section{IV. $1^{\text {st }}$ configuration: collimating lens + curved pillar}

In this section, we investigate the use of a collimating lens followed by a curved pillar to deflect the beam to the back of the port-plug. The capillaries of the pillar are exactly the continuity of the capillaries of the collimating lens, thus they have the same diameter, see FIG. 3d. In order to compare properly the different transmissions for the 3 beams arriving in the boxes, we used three times the same collimating lens with a focal length of $37 \mathrm{~mm}$, input and output diameters respectively of $5 \mathrm{~mm}$ and $9.7 \mathrm{~mm}$ and a capillary diameter of $50 \mu \mathrm{m}$.

We focused the simulations on the third beam which has the worst transmission by far due to the huge deflection angle. A transmission inferior to $0.2 \%$ is obtained for X-rays between 1 $\mathrm{keV}$ and $15 \mathrm{keV}$, see FIG. 4. On the one hand this poor transmission is due to the design of the boxes and associated LoS geometry. On the other hand, a great loss of transmission is also due to the change of curvature radius of the monocapillaries between the collimating lens and the pillar. This change of curvature radius makes a great part of the $\mathrm{X}$-rays have a first reflection in the pillar with a huge angle, often superior to the critical angle (as introduced in FIG. 1) at the considered energy.

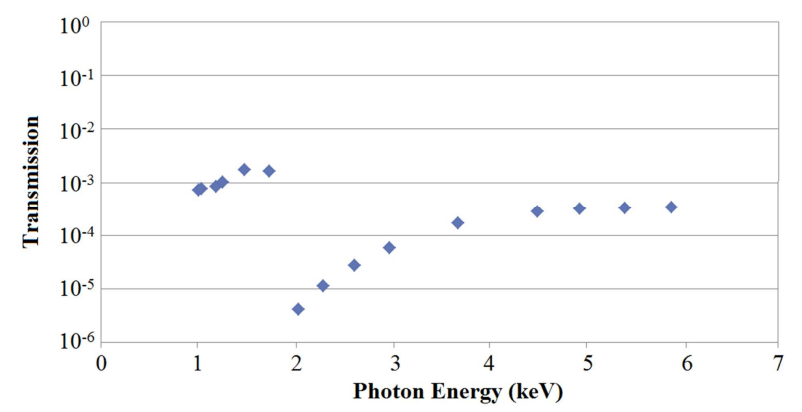

FIG. 4: Transmission vs photon energy for the beam 3

\section{V. $2^{\text {nd }}$ configuration: bench of monocapillaries}

In a second time, and in order to avoid the disadvantageous effect of the collimating lens on the transmission, a bench of separated monocapillaries has been studied with curvature radius defined independently. We focused the simulation on 3 capillaries by beam as presented in FIG. 5.
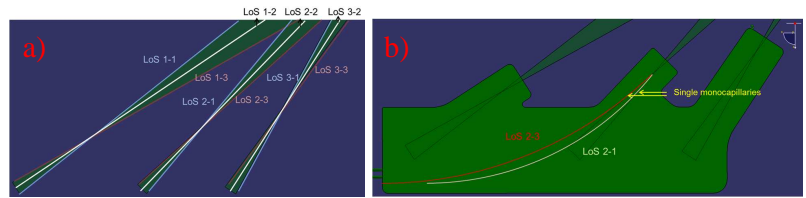

FIG. 5: a) Schema of the lines of sight (LoS) for the 3 beams and b) 2 examples of the monocapillaries studied 
The results as presented in FIG. 6 show that the transmission can be greatly improved by designing such monocapillaries. Indeed, we can win up to one order of magnitude for the $3^{\text {rd }}$ beam. Even if we never reach the ideal $1 \%$ target for the $3^{\text {rd }}$ beam either, the gain (for the LoS 3-2 we increase from $3.10^{-4}$ to $10^{-3}$ ) comes from this change of configuration. X-ray propagation at the output of the curved monocapillaries is not rigorously horizontal. Indeed photons can have an small angle compared with the horizontal up to few mrad. So with great lengths up to the detectors, it can become difficult to infer where the rays come from. Thus the addition of straight monocapillaries at the output of the monocapillaries appears necessary to transport X-rays up to the back of the box. However several $\operatorname{LoS}(1-1,1-2,1-3,2-3)$ do not need a straight capillary because the curved monocapillaries already end at the back of the box.

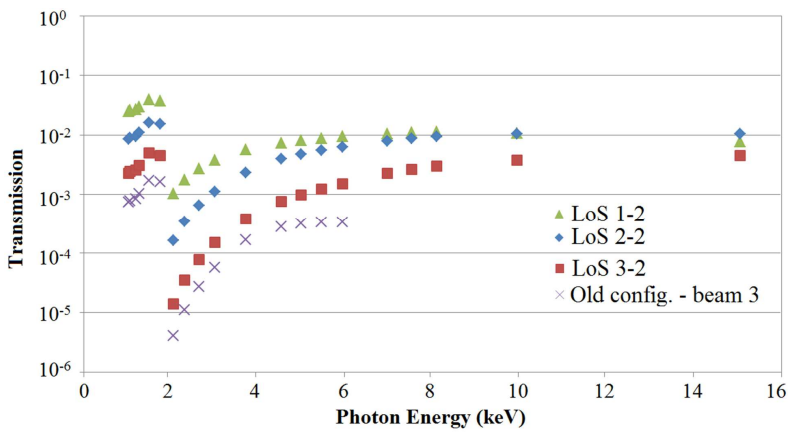

FIG. 6: Transmission of different LoS using the bench of monocapillaries

Transmission studies for the different LoS and the influence of the capillary diameters are reported in FIG. 7. For intermediate capillary diameters $(\sim 20 \mu \mathrm{m})$ the transmission of beam $n^{\circ} 1$ is quite sufficient, i.e. above $1 \%$ for energies $<2 \mathrm{keV}$ and $>4 \mathrm{keV}$. We lose one order of magnitude for the beam $n^{\circ} 2$ compared with beam $\mathrm{n}^{\circ} 1$, but the transmission remains above $0.1 \%$ in this energy range. The straight monocapillaries are taken into account for the LoS 2-2 and 3-1 and have a length respectively of 103 $\mathrm{mm}$ and $756 \mathrm{~mm}$. The LoS 3-1 corresponds to the highest deflection angle $60.8^{\circ}$ and thus to the lowest transmission overall. A new strategy is proposed to that particular beam in order to improve its transmission.

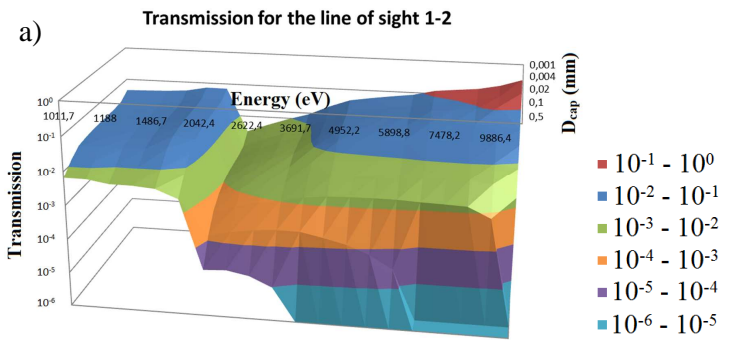

b)

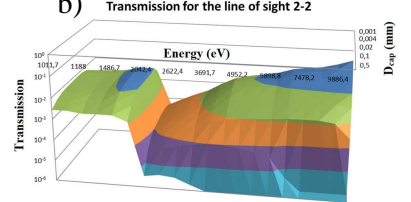

c) Transmission for the line of sight $3-1$

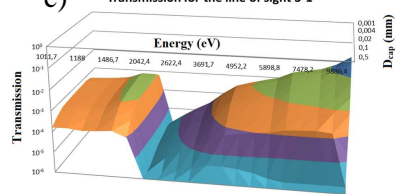

FIG. 7: Transmission of the $\operatorname{LoS}$ a) 1-2 b) 2-2 c) 3-1, with curved monocapillaries of a) $448 \mathrm{~mm}$ b) $1115 \mathrm{~mm}$ c) $989 \mathrm{~mm}$, curvature radius of a) $779 \mathrm{~mm} \mathrm{b)} 1452 \mathrm{~mm}$ c) $931 \mathrm{~mm}$, deflection angle of a) $33^{\circ}$ b) $44^{\circ}$ c) $61^{\circ}$, and straight pillars of a) no straight pillar

b) $103 \mathrm{~mm}$ c) $756 \mathrm{~mm}$

Unlike the previous configurations, we will allow from now the monocapillaries to have a non-horizontal output in order to decrease the global X-ray deflection, and thus to increase the transmission. The output angle is defined as the angle between the output beam and the horizontal axis. The range of studied output angles is between the 2 extreme cases with a horizontal output (previous studied cases) and an output at the back of the box with the highest possible angle $\left(14.64^{\circ}\right)$ as illustrated in FIG. 8a. As expected, the transmission increases with the output angle, up to one order of magnitude for the most favorable case, see FIG. 8b-c.
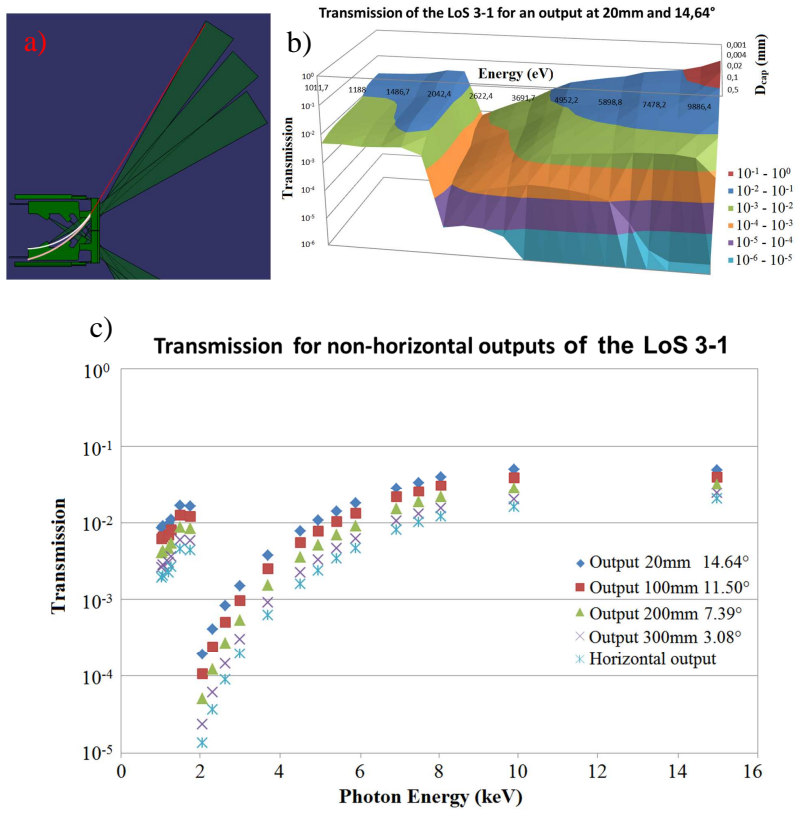

FIG. 8: a) Schema of the two extreme cases with horizontal and oblique output. b) Transmission of the LoS 3-1 in the most favorable case with an output angle of $14.64^{\circ}$. c) Variation of the transmission with the output angle

As conclusion the bench of monocapillary is found to be the best solution so far to transport SXR information during the ITER D$\mathrm{T}$ phase. It has also been shown that some adaptation of the port plug design internal geometry could increase further the transmission of the polycapillaries, in particular for the most unfavorable LoS in terms of SXR transmission.

\section{REFERENCES}

1 D. Mazon et al, "Polycapillary lenses for Soft-X-ray transmission: Model, comparison with experiments and potential application for tomographic measurements in tokamaks," Nuclear Instruments and Methods in Physics Research B, 2015.

${ }^{2}$ X. Lin, et al. A Matlab programming for simulation of X-ray capillaries Appl. Math. Comput., 172 (2006), pp. 188-197. 